\title{
An objective function to address production sequencing with minimal tooling replacements
}

\author{
P. R. MCMULLEN* \\ Babcock Graduate School of Management, Wake Forest University, \\ Winston-Salem, North Carolina 27109, USA
}

(Revision received September 2005)

\begin{abstract}
A strategy is presented to obtain production sequences resulting in minimal tooling replacements. An objective function is employed to distribute the tool wear as evenly as possible throughout the sequence. This objective function is an extension of Miltenburg's earlier work (1989) concerned with obtaining production sequences while evenly distributing the satisfaction of demand. Smaller problems are solved to optimality, while larger problems are solved as close as possible to optimality. The production sequences are simulated to estimate required tooling replacements. The methodology presented here consistently results in fewer tooling replacements when compared with earlier published work (McMullen et al. 2002, McMullen 2003).
\end{abstract}

Keywords: Optimization; Search; Heuristic; Simulated annealing

\section{Introduction}

Scheduling has received a great deal of attention in terms of production research. The objectives of production scheduling are many: minimization of idle time, minimization of work-in-process inventory, minimization of overtime, etc. On the other hand, production scheduling dedicated to minimization of tooling replacements has seen a minimal amount of attention.

When items are manufactured, the resources used in manufacturing are subject to wear. Consider a steel-stamping application. After several units have been stamped into their desired form, the tooling wears down and will need repair and/or replacement. Earlier research efforts (McMullen et al. 2002, McMullen 2003) have shown that sequencing production in the appropriate manner can reduce the requirement to repair or replace the tooling. Subsequently, costs can be reduced by properly sequencing production when tool wear is a source of concern. Another, but more general example is that of tyre wear. Most automobile owners are encouraged to have their tyres rotated every 3000 miles. Tyre rotation permits the tyres to 'wear' in new areas and therefore more evenly distribute the tyre wear, ultimately extending the life of the car's tyres.

While the tyre rotation example is simple to understand, other applications may be more difficult to understand. This may especially be the case when complicating

*Email: patrick.momullen@mba.wfu.edu 


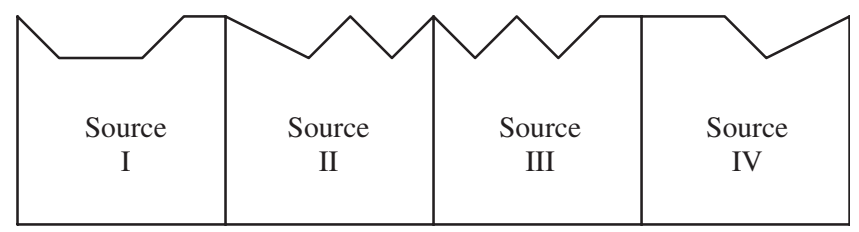

Figure 1. Uneven distribution of wear.

factors persist: multiple items are manufactured through a single production line; multiple manufacturing resources are required for each item; there are 'just in time' (JIT) objectives, etc. Given such real-world complicating factors, the challenge of finding production sequences resulting in evenly distributed tool wear, with the subsequent minimization of tooling replacement, is an objective that, in the opinion of the author, has not seen enough attention in the literature. The fact that sequencing to minimize tooling replacement is a complicated issue, along with its importance, is the motivation for this research effort.

With this research effort, background information is provided relative to the importance of evenly distributing tool wear. An objective function is carefully developed to illustrate how one can succeed in production sequencing that clearly optimizes the distribution of tool wear. This objective function, and associated methodology, is then deployed to several test problems from the literature. The production sequences obtained from this methodology are then simulated to determine the expected number of tooling replacements. The results from the production simulation are then compared to the results of production simulation from prior research efforts addressing the same problem. The relative superiority of the present methodology is then detailed. Limitations of this research effort are detailed, and opportunities for future research are then discussed.

\section{Importance of evenly distributing tool wear}

Consider the following scenario: three unique items (A, B, and C) need to be manufactured. Demand for item A is 4 units; demand for B is three units, while demand for item $\mathrm{C}$ is two units. Additionally, four resources are needed to manufacture each unique item. Each production resource is subject to wear, in the form of cutting, machining, etc. These resources are referred to as 'wear sources' hereafter. Each unique item imposes an expected degree of wear upon each of the wear sources, and with this expectation, comes some degree of variation. Subsequently, the degree of wear that each unique item imposes on each wear source can be thought of as a stochastic variable. After the production sequences are repeated several times, the wear source's ability to machine will deteriorate and require replacement and/or repair. Figure 1 below shows a scenario in which the wear is unevenly distributed (especially regarding sources II and III), while figure 2 shows a scenario in which the wear is more evenly distributed (all four sources have approximately the same wear).

Past research has proven that appropriate production sequencing can more evenly distribute wear, and subsequently, minimize the amount of replacement 


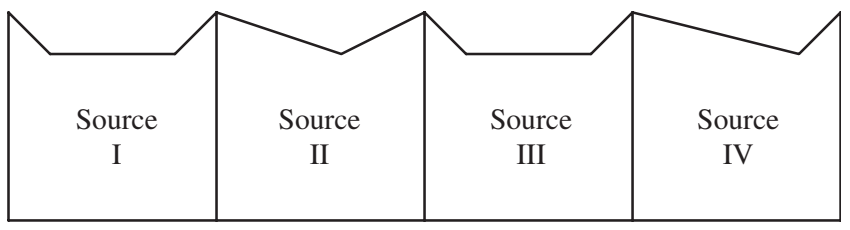

Figure 2. Even distribution of wear.

and/or repair of tooling. As a result of this relationship between distribution of tool wear and need for replacement and/or repair, it is desired to find production sequences which do the best possible job of sequencing to evenly distribute tool wear. Past research by McMullen et al. (2002) and McMullen (2003) suggested that additional opportunities exist for improving the ability to more evenly distribute tool wear. The present research effort is concerned with providing a mathematical objective which results in an optimal degree of even distribution of tool wear.

\section{Methodology}

This section details the specifics of the research methodology. First the problem formulation is formally presented. An example is then presented to clarify the methodology. The issue of optimization of the objective function value is then discussed.

\subsection{Problem formulation}

As stated earlier, this research extends the earlier efforts of Miltenburg (1989). Miltenburg's earlier work was concerned with production sequencing resulting in the unique items being produced in direct proportion to their respective levels of demand. The extrapolation here, from Miltenburg's earlier effort, is to extend the Miltenburg mindset to find a sequence such that the wear across all wear sources occurs in a linear fashion, in direct proportion to the total wear for each source. The following definitions are stated to assist with the methodological development:

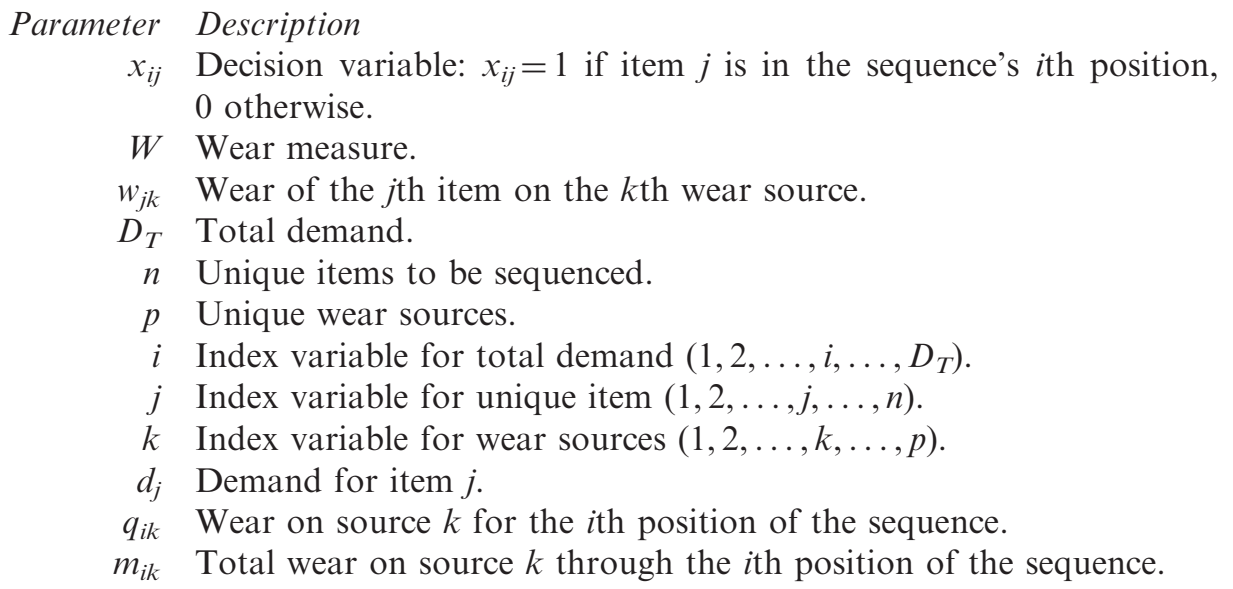


It is noted that the only variables under the direct control of the decision-maker (the decision variables) are the binary variables concerned with the unique item's placement into the production sequence (the $x_{i j}$ values). These values are chosen such that the wear measure value $(W)$ is minimized. With this in mind, it is desirable that either of the following general objective functions be minimized:

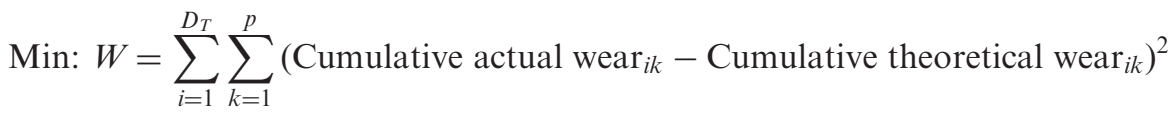

Min: $W=\sum_{i=1}^{D_{T}} \sum_{k=1}^{p} \mid$ Cumulative actual wear - Cumulative theoretical wear $\mid$

The first of the above objective functions (1) seeks to minimize the sum of squared differences between the cumulative actual wear and cumulative theoretical wear associated with all $p$ wear sources and $D_{T}$ sequence positions. The squaring of each difference is done for two reasons: first to eliminate the negative sign; second to amplify differences - analogous to a variance calculation. The second of these objective functions (2) desires to minimize the sum of differences between cumulative actual wear and cumulative theoretical wear. For equation (2), absolute values are used to eliminate a negative sign. Either of these approaches can be considered appropriate (as stated by Miltenburg 1989), but for the remainder of this paper, objective function (1) will be used throughout for two reasons. First, the author considers the squaring of wear differences an important feature of this approach, so that large gaps between actual and theoretical wear are amplified. Second, Miltenburg's seminal paper (1989) was developed with respect to the squaring of differences, analogous to equation (1). As such, the author wishes to preserve the structure of the earlier Miltenburg effort (1989).

This objective can be seen graphically (as a comparison of cumulative actual wear and cumulative theoretical wear), in a generalized form, via figure 3 .

Figure 3 shows that the straight-line represents the cumulative theoretical wear-a linear representation of even tool wear throughout the sequence. The 'stepped' line shows cumulative actual wear throughout the sequence. This objective function is minimized when the 'stepped' line is as close as possible to the straight line.

For the cumulative theoretical wear component, the vertical axis component is related to the $i$ th position in the sequence (where $i$ is the independent variable) via the equation:

$$
\text { Cumulative theoretical wear }=\left(\frac{i}{D_{T}}\right) m_{D_{T} k}, \quad \forall k
$$

The cumulative actual wear component is simply the value of $m_{i k}$, which is defined by:

$$
m_{i k}=\sum_{g=1}^{i} q_{g k}, \quad \forall i, k \text { combinations }
$$




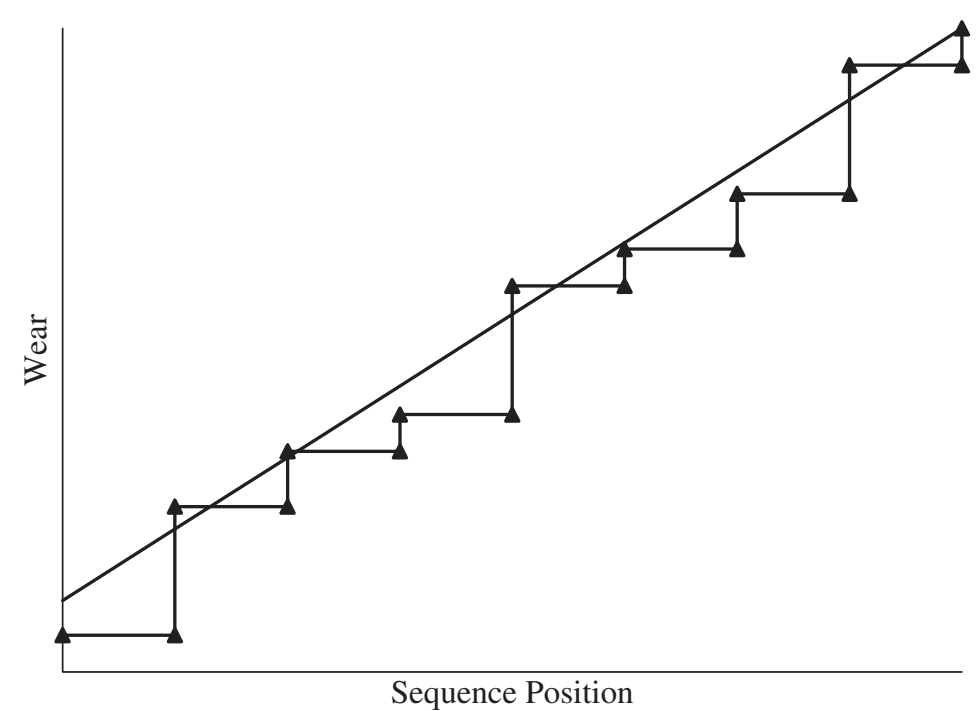

Figure 3. Graphical representation of wear measure calculation.

where:

$$
q_{i k}=\sum_{j=1}^{n} x_{i j} w_{j k}, \quad \forall i, k \text { combinations }
$$

Combining these components into a single mathematical programming formulation yields the following:

$$
\text { Min: } W=\sum_{i=1}^{D_{T}} \sum_{k=1}^{p}\left(m_{i k}-\left(\frac{i}{D_{T}}\right)\left(m_{D_{T} k}\right)\right)^{2},
$$

subject to:

$$
\begin{aligned}
& \sum_{i=1}^{D_{T}} x_{i j}=d_{j}, \quad \forall j \\
& \sum_{j=1}^{n} x_{i j}=1, \quad \forall i
\end{aligned}
$$

The constraint in equation (7) guarantees that total demand for each unique item is met, while the constraint in equation (8) guarantees that each of the $D_{T}$ sequence positions contain exactly one item. The objective function can also be expressed in terms of the decision variables via the following:

$$
\operatorname{Min}: W=\sum_{i=1}^{D_{T}} \sum_{k=1}^{p}\left(\sum_{g=1}^{i}\left(\sum_{j=1}^{n} x_{i j} w_{j k}\right)-\left(\frac{i}{D_{T}}\right)\left(\sum_{g=1}^{D_{T}}\left(\sum_{j=1}^{n} x_{i j} w_{j k}\right)\right)\right)^{2}
$$


This objective function, of course, is also subject to the same constraints already shown in equations (7) and (8). The objective function in equation (9) articulates the same as the objective function in equation (6). While the objective function in equation (6) is perhaps more understandable, the presentation of the objective function in equation (9) is only made in the interest of relating the formulation directly to the decision variables.

\subsection{Example problem}

To illustrate the construction of the formulation presented above, a detailed example is provided. Consider the example problem described in the previous section. There are three unique items: item $\mathrm{A}$ has demand of four units $\left(d_{1}=4\right)$, item $\mathrm{B}$ has three units $\left(d_{2}=3\right)$ demanded, and item $\mathrm{C}$ has two units $\left(d_{3}=2\right)$ demanded. This is a total demand of nine units $\left(D_{T}=d_{1}+d_{2}+d_{3}=9\right)$. These three unique items will be processed over four sources. Subsequently, there are four sources of tool wear. The expected degree of wear for each item across each wear source is detailed in table 1.

These wear values are used as the $w_{j k}$ values for the formulation. For example, the tool wear for item B on wear source $4\left(w_{24}\right)$ is 7 units.

Consider the sequence ABCABACBA. The assignment of binary decision variables ( $x_{i k}$ values) for this particular sequence is detailed in table 2 .

Note that for this sequence, the constraints detailed in equations (7) and (8) are satisfied. The $q_{i k}$ values associated with this sequence are detailed in table 3 .

For example, the value for the third wear source in the fifth position $\left(q_{53}\right)$ is determined by $q_{53}=x_{51} w_{13}+x_{52} w_{23}+x_{53} w_{32}=(0)(3)+(1)(6)+(0)(3)=6$.

Table 1. Wear data for example problem.

\begin{tabular}{lcccc}
\hline Item & Wear 1 & Wear 2 & Wear 3 & Wear 4 \\
\hline A & 2 & 2 & 3 & 2 \\
B & 6 & 5 & 6 & 7 \\
C & 4 & 3 & 3 & 3 \\
\hline
\end{tabular}

Table 2. Sequence for example problem.

\begin{tabular}{lccc}
\hline Position & Item $1\left(x_{i 1}\right)$ & Item $2\left(x_{i 2}\right)$ & Item $3\left(x_{i 3}\right)$ \\
\hline $1\left(x_{1 k}\right)$ & 1 & 0 & 0 \\
$2\left(x_{2 k}\right)$ & 0 & 1 & 0 \\
$3\left(x_{3 k}\right)$ & 0 & 0 & 1 \\
$4\left(x_{4 k}\right)$ & 1 & 0 & 0 \\
$5\left(x_{5 k}\right)$ & 0 & 1 & 0 \\
$6\left(x_{6 k}\right)$ & 1 & 0 & 0 \\
$7\left(x_{7 k}\right)$ & 0 & 0 & 1 \\
$8\left(x_{8 k}\right)$ & 0 & 1 & 0 \\
$9\left(x_{9 k}\right)$ & 1 & 0 & 0 \\
\hline
\end{tabular}


Table 3. Wear associated with example problem.

\begin{tabular}{lcccc}
\hline Position & Wear 1 $\left(q_{i 1}\right)$ & Wear 2 $\left(q_{i 2}\right)$ & Wear 3 $\left(q_{i 3}\right)$ & Wear 4 $\left(q_{i 4}\right)$ \\
\hline $1\left(q_{1 k}\right)$ & 2 & 2 & 3 & 2 \\
$2\left(q_{2 k}\right)$ & 6 & 5 & 6 & 7 \\
$3\left(q_{3 k}\right)$ & 4 & 3 & 3 & 3 \\
$4\left(q_{4 k}\right)$ & 2 & 2 & 3 & 2 \\
$5\left(q_{5 k}\right)$ & 6 & 5 & 6 & 7 \\
$6\left(q_{6 k}\right)$ & 2 & 2 & 3 & 2 \\
$7\left(q_{7 k}\right)$ & 4 & 3 & 3 & 3 \\
$8\left(q_{8 k}\right)$ & 6 & 5 & 6 & 7 \\
$9\left(q_{9 k}\right)$ & 2 & 2 & 3 & 2 \\
\hline
\end{tabular}

Table 4. Actual cumulative wear associated with example problem.

\begin{tabular}{lcccc}
\hline Position & Wear 1 $\left(m_{i 1}\right)$ & Wear 2 $\left(m_{i 2}\right)$ & Wear 3 $\left(m_{i 3}\right)$ & Wear 4 $\left(m_{i 4}\right)$ \\
\hline $1\left(m_{1 k}\right)$ & 2 & 2 & 3 & 2 \\
$2\left(m_{2 k}\right)$ & 8 & 7 & 9 & 9 \\
$3\left(m_{3 k}\right)$ & 12 & 10 & 12 & 12 \\
$4\left(m_{4 k}\right)$ & 14 & 12 & 15 & 14 \\
$5\left(m_{5 k}\right)$ & 20 & 17 & 21 & 21 \\
$6\left(m_{6 k}\right)$ & 22 & 19 & 24 & 23 \\
$7\left(m_{7 k}\right)$ & 26 & 22 & 27 & 26 \\
$8\left(m_{8 k}\right)$ & 32 & 27 & 33 & 33 \\
$9\left(m_{9 k}\right)$ & 34 & 29 & 36 & 35 \\
\hline
\end{tabular}

Table 5. Theoretical cumulative wear associated with example problem.

\begin{tabular}{lrrrr}
\hline Position & Wear 1 & Wear 2 & Wear 3 & Wear 4 \\
\hline 1 & 3.78 & 3.22 & 4.00 & 3.89 \\
2 & 7.56 & 6.44 & 8.00 & 7.78 \\
3 & 11.33 & 9.67 & 12.00 & 11.67 \\
4 & 15.11 & 12.89 & 16.00 & 15.56 \\
5 & 18.89 & 16.11 & 20.00 & 19.44 \\
6 & 22.67 & 19.33 & 24.00 & 23.33 \\
7 & 26.44 & 22.56 & 28.00 & 27.22 \\
8 & 30.22 & 25.78 & 32.00 & 31.11 \\
9 & 34.00 & 29.00 & 36.00 & 35.00 \\
\hline
\end{tabular}

This logic is repeated for all $i$ and $k$ combinations. The $m_{i k}$ values associated with this sequence are detailed in table 4.

For example, the cumulative wear for the third wear source in the fifth position of the sequence $\left(m_{53}\right)$ is simply a running sum of the third column $(k=3)$ of the fifth row $(i=5)$. Mathematically, this is as follows: $m_{53}=q_{13}+q_{23}+q_{33}+q_{43}+q_{53}=$ $3+6+3+3+6=21$. Table 5 below shows the theoretical wear through the $i$ th position in the sequence. 
These values are determined via equation (3). For the presented example, the cumulative theoretical wear for the fifth position of wear source 3 is $(5 / 9) \cdot 36$, which is 20 .

The wear measure for this example problem is the sum of the squared differences between the $m_{i k}$ values and their corresponding values from table 5. For example, the wear source 3 for the fifth position in the sequence contributes $(21-20)^{2}$ to the objective function value. For the entire sequence the wear measure is $W=36.6667$. Figure 4 shows, for each of the four wear sources for this example problem, the cumulative actual tool wear in relation to the cumulative theoretical tool wear. Actually, two different sequences are plotted. The sequence with the $\boldsymbol{\Delta}$ symbol is the ABCABACBA sequence-the sequence developed for this example. The sequence with the symbol is the AAAABBBCC sequence, which was arbitrarily chosen. The theoretical cumulative wear for both of these sequences is the straight line.

Again, the wear measure value for the ABCABACBA sequence is $W=36.6667$. The wear measure for the arbitrarily chosen AAAABBBCC sequence is $W=354.1111$. Note how the 'stepped-line' for the ABCABACBA sequence $(\boldsymbol{\Delta})$ is typically closer to the straight line than the AAAABBBCC function ( $\boldsymbol{\square})$. This provides a graphic explanation to the fact that tool wear is more evenly distributed for the ABCABACBA $(\boldsymbol{\Delta})$ sequence as compared to the AAAABBBCC sequence $(\boldsymbol{\square})$.

In fact, the ABCABACBA sequence provides the minimum wear measure of all possible sequence permutations for this example problem.

\subsection{Optimization of objective function}

As just stated, the sequence used for the example problem yields the minimum wear measure of all possible sequences. The number of possible sequences is determined via the following:

$$
\text { Possible sequences }=\frac{\left(\sum_{j=1}^{n} d_{j}\right) !}{\prod_{j=1}^{n}\left(d_{j} !\right)}
$$

For the example problem, the number of possible sequences is $(4+3+2)$ !/ $((4 !)(3 !)(2 !))=1260$. For a smaller problem such as this, enumeration of all possible permutations is possible. For only slight increases in problem size, however, direct enumeration becomes intractable. Another complicating factor is that although the objective functions presented in equations (6) and (9) show quadratic and convex properties (Nemhauser and Wolsey 1988, Pinedo 2001), solution to optimality via mathematical programming is problematic due to the fact that the binary decision variables induce a discontinuous objective function, which frequently renders commercial software solvers into a state which may or may not yield an optimal solution to a problem of the sort addressed here (Fourer et al. 2002). As such, a heuristic approach is used here to find near-optimal solutions for the larger problems - enumeration is still used for the smaller problems. In other words, problems of this type are so enormous, that the author feels justification in using a modern search heuristic to gain a hopefully near-optimal solution without the 
(a)

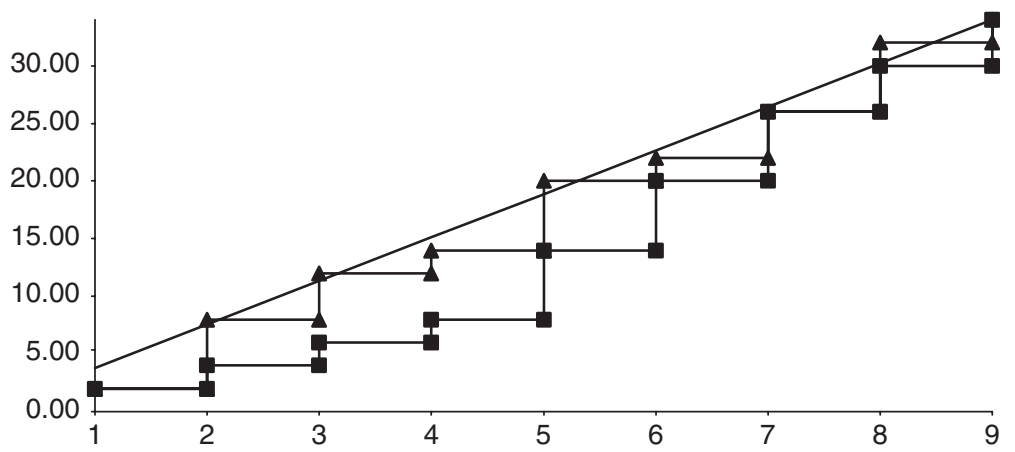

(b)

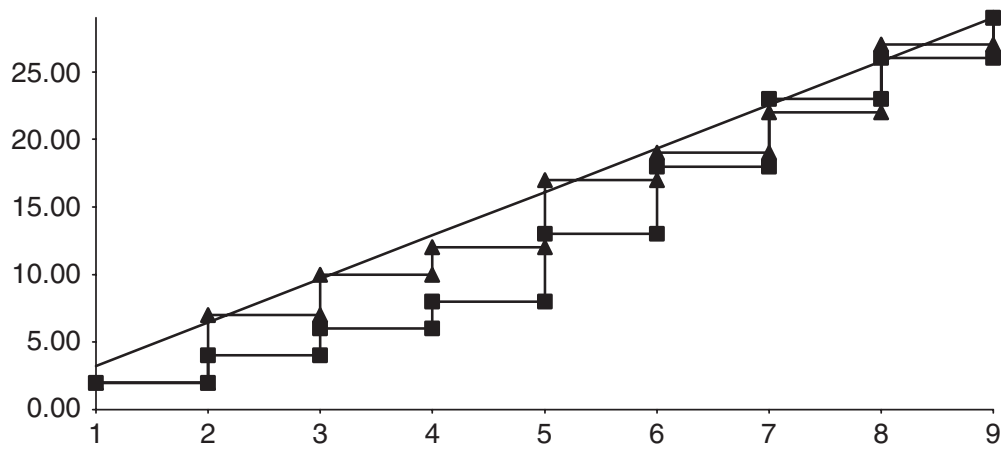

(c) 35.00

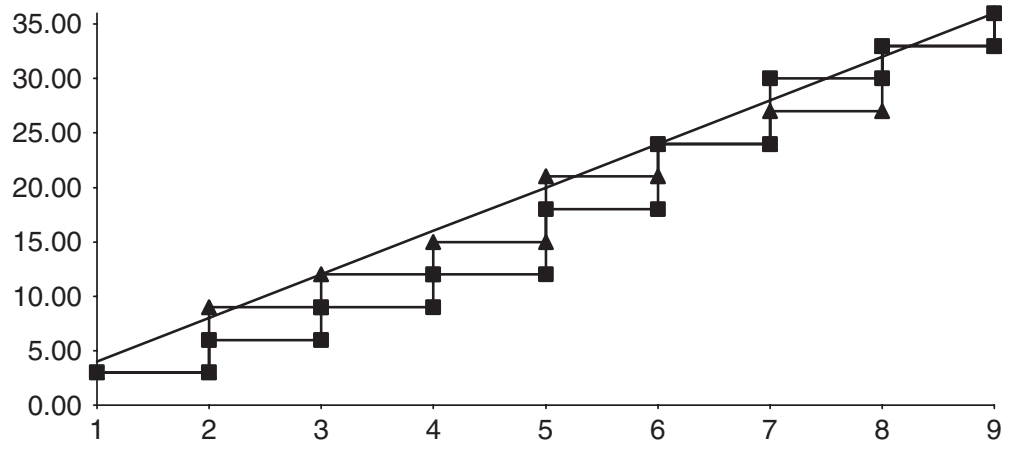

(d) 35.00

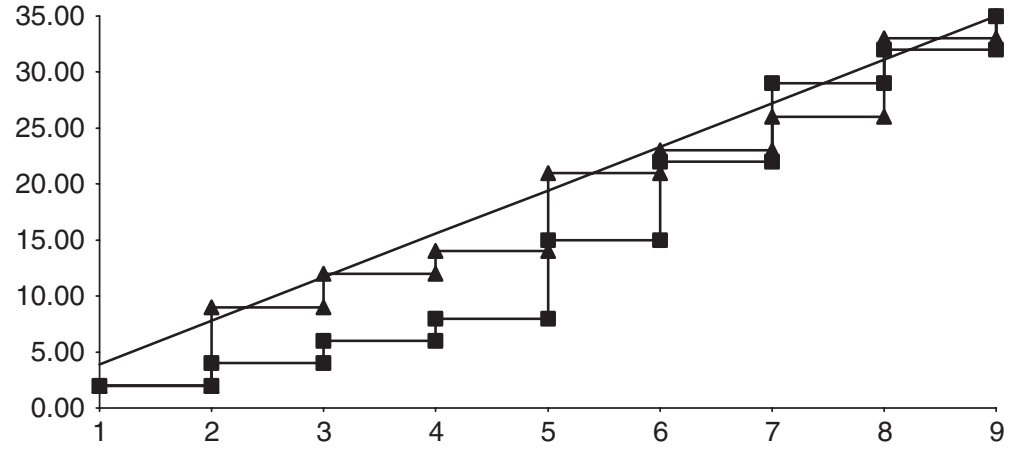

Figure 4. (a) Wear source 1; (b) wear source 2; (c) wear source 3; (d) wear source 4. 
simultaneous rigor of a computationally expensive optimal approach. Ding et al. (1993) and Kubiak et al. (1991) offer sequencing heuristics for the Miltenburg objective function, but they are not used here due to the complicating factors associated with the modification of Miltenburg's methodology. Hence, another heuristic approach must be employed.

The heuristic of choice for this research effort is simulated annealing. Simulated annealing itself is not a focus of this research, only a means of obtaining a desirable objective function value, and the interested reader is referred to the seminal work of Kirkpatrick et al. (1983) and Eglese (1990) for more information. Here, the simulated annealing heuristic works by first randomly generating an initial feasible solution. The sequence is then modified via pairwise swaps of unique sequence members. The objective function is determined for the new sequence. If the objective function value is an improvement over the objective function value of the current solution, the new solution becomes the current solution. If the objective function value of the new solution is inferior to that of the current solution, the new solution will still replace the current solution if and only if some probabilistic condition is met. This probabilistic condition is referred to as the Metropolis Criterion (Metropolis et al. 1953), and is the unique feature of simulated annealing. Relatively inferior solutions are used, with a small probability, to replace relatively superior solutions with the intent of relocating to a new locus of the feasibility continuum with the hope that these new solutions will ultimately yield a new global optimal condition (or a near-global optimal condition).

\section{Experimentation}

Simply finding sequences providing minimal values of $W$ does not give the researcher a licence to claim superior results. These sequences must be tested on several problems, and these sequences must be applied to some real-world condition, so that generalized findings can be articulated. As such, the methodology described above is applied to a problem set from the literature, and these sequences are then simulated to determine the number of required tooling replacements.

\subsection{Problem sets}

There exists in the literature (McMullen et al. 2002) a set of 11 problems dedicated to this issue of even distribution of tool wear. Table 6 details these problems.

The table above shows for each problem in the set, the number of wear areas $(p)$, the product mix, the range of tool wear over each wear source and the number of possible sequences (feasible solutions). For the smaller problems (1 to 6), the optimal solution in terms of the wear measure $(W)$ was found via enumeration of all feasible solutions. For the larger problems (7 to 11) simulated annealing was used to find the solution with the minimal wear measure value.

\subsection{Production simulation}

A production simulation of each obtained sequence is performed to determine the number of changeovers required of each sequence. The simulation is quite simple. 
Table 6. Problem set detail.

\begin{tabular}{|c|c|c|c|c|}
\hline Problem & $\begin{array}{c}\text { Wear } \\
\text { areas }(p)\end{array}$ & Product-mix & $\begin{array}{l}\text { Range of } \\
\text { tool wear }\end{array}$ & $\begin{array}{l}\text { Possible } \\
\text { sequences }\end{array}$ \\
\hline 1 & 3 & $\begin{array}{l}\mathrm{A}(1), \mathrm{B}(1), \mathrm{C}(1), \mathrm{D}(1), \mathrm{E}(1), \\
\mathrm{F}(1), \mathrm{G}(1), \mathrm{H}(1)\end{array}$ & $1-3$ & 40,320 \\
\hline 2 & 3 & $\begin{array}{l}\mathrm{A}(1), \mathrm{B}(1), \mathrm{C}(1), \mathrm{D}(1), \mathrm{E}(1) \\
\mathrm{F}(1), \mathrm{G}(1), \mathrm{H}(1), \mathrm{I}(1)\end{array}$ & $1-3$ & 362,880 \\
\hline 3 & 3 & $\begin{array}{l}\mathrm{A}(1), \mathrm{B}(1), \mathrm{C}(1), \mathrm{D}(1), \mathrm{E}(1), \mathrm{F}(1), \\
\quad \mathrm{G}(1), \mathrm{H}(1), \mathrm{I}(1), \mathrm{J}(1)\end{array}$ & $1-7$ & $3,628,800$ \\
\hline 4 & 4 & $\mathrm{~A}(4), \mathrm{B}(3), \mathrm{C}(2), \mathrm{D}(1), \mathrm{E}(1), \mathrm{F}(1)$ & $1-8$ & $1,663,200$ \\
\hline 5 & 4 & $\mathrm{~A}(2), \mathrm{B}(2), \mathrm{C}(2), \mathrm{D}(2), \mathrm{E}(2)$ & $1-10$ & 113,400 \\
\hline 6 & 3 & $\mathrm{~A}(3), \mathrm{B}(3), \mathrm{C}(2), \mathrm{D}(2), \mathrm{E}(2)$ & $1-25$ & $1,663,200$ \\
\hline 7 & 5 & $\begin{array}{l}\mathrm{A}(4), \mathrm{B}(4), \mathrm{C}(3), \mathrm{D}(3), \mathrm{E}(2), \\
\mathrm{F}(2), \mathrm{G}(1), \mathrm{H}(1)\end{array}$ & $1-9$ & $2.993(10)^{13}$ \\
\hline 8 & 3 & $\mathrm{~A}(12), \mathrm{B}(3), \mathrm{C}(2), \mathrm{D}(2), \mathrm{E}(1)$ & $1-25$ & $219,629,600$ \\
\hline 9 & 3 & $\mathrm{~A}(12), \mathrm{B}(3), \mathrm{C}(2), \mathrm{D}(2), \mathrm{E}(1)$ & $1-15$ & $219,629,600$ \\
\hline 10 & 3 & $\mathrm{~A}(4), \mathrm{B}(4), \mathrm{C}(4), \mathrm{D}(4), \mathrm{E}(4)$ & $1-15$ & $3.0554(10)^{11}$ \\
\hline 11 & 3 & $\mathrm{~A}(4), \mathrm{B}(4), \mathrm{C}(4), \mathrm{D}(4), \mathrm{E}(4)$ & $1-30$ & $3.0554(10)^{11}$ \\
\hline
\end{tabular}

Each time an item in a sequence is processed, the level of available tooling for each source $\left(\mathrm{Tool}_{k}\right)$ is adjusted according to the following relationship:

$$
\begin{gathered}
\operatorname{Tool}_{k}=\operatorname{Tool}_{k}-\left(w_{j k}+\text { variant }\right), \quad \forall k \\
\text { where variant }=w_{j k} * c v * z \\
\text { if variant } \leq-0.2 * w_{j k}, \text { then variant }=-0.2 * w_{j k}
\end{gathered}
$$

The quantity 'variant' is included in the above to quantify stochastic behaviour to the degree of tool wear that occurs on each source of wear. After initialization, each item $i$ is processed in the sequence, and each wear source's level of tooling is adjusted according to the formulae above. A check is then made to determine $\mathrm{Tool}_{k}$ has fallen below the value of zero. If the amount of available tooling has fallen below the value of zero, then the number of replacements is incremented by one. If a tooling replacement is required, the value of available tooling for all sources is reset to its upper threshold level. For the experimentation here, the value of the threshold is 50, and each simulated sequence is replicated 2000 times to capture a long-term measure of replacements. For each sequence, the simulation run is repeated 25 times so that a reasonable estimate of required replacements is possible. These 25 replications for each sequence are then repeated for three different co-efficients of variation $(\mathrm{cv})$ : $0.05,0.15,0.25$ - these quantities are included to provide the modeller control over the level of variation in simulated tool wear (McMullen 2003). A total of 75 simulated runs for each production sequence are evaluated.

\subsection{Computational experience}

Attainment of sequences via the objective function, and execution of the production simulation were conducted via programs written with the 
Borland $\mathrm{C}++$ Builder compiler. These programs were run using the Microsoft Windows XP operating system, on a Pentium 4 processor with a speed of $2.26 \mathrm{GHz}$.

\subsection{Research question}

It is desired to determine whether or not the methodology presented here compares favourably to results from earlier research efforts. In this context, 'results' imply the number of replacements required for each sequence. To make this comparison, the average tooling replacements obtained from the methodology presented here are measured against the average tooling replacements associated with the best of the performers from the earlier research (McMullen et al. 2002) and McMullen (2003). Several objective functions were explored from two earlier publications. The 'best' performer for each of the 11 problems in the problem set is then used for comparison with the results associated with the methodology presented here. The mean replacements associated with the best performer from the earlier research are referred to as $\mu_{1}$. The replacements associated with the methodology presented here are referred to as $\mu_{2}$. The research question then becomes: 'Does the methodology presented here outperform, in terms of mean replacements, the best performing methodology from earlier work in this area?' This question can also be stated mathematically as follows:

$$
\begin{array}{ll}
\mathrm{H}_{0}: & \mu_{2}=\mu_{1} \\
\mathrm{H}_{1}: & \mu_{2}<\mu_{1}
\end{array}
$$

This research question is addressed via a comparison of means $t$-test for each of the 11 problems in the problem set.

\section{Experimental results}

Table 7 shows the results of the replacements associated with the sequences obtained from the earlier research compared with the replacements associated with the research presented here.

For each problem in the problem set, the mean replacements and standard deviations are provided for both the earlier research $\left(\mu_{1}\right.$ and $\left.s_{1}\right)$ and the research presented here $\left(\mu_{2}\right.$ and $\left.s_{2}\right)$. Also provided for each problem are the pooled standard deviation $\left(s_{p}\right)$, the $t$-statistic $(t)$ and the $p$-value.

For problem 1, the presented research results in fewer average replacements than does the best of the earlier research, but this difference in replacements is not significant to any reasonable degree. For problem 2, the best of the previous research outperforms the present research, and this difference is significant, although the difference in means is only 2.0 replacements for every 2000 sequence replications. For all other problems, the presented research results in fewer replacements than does the best of the earlier research, and these differences are significant at the $\alpha=0.0001$ level. 
Table 7. Performance comparison of earlier research with current research.

\begin{tabular}{lrrrrrrr}
\hline Problem & \multicolumn{1}{c}{$\mu_{1}$} & \multicolumn{1}{c}{$s_{1}$} & \multicolumn{1}{c}{$\mu_{2}$} & \multicolumn{1}{c}{$s_{2}$} & \multicolumn{1}{c}{$s_{\mathrm{p}}$} & \multicolumn{1}{c}{$t$} & $p$-value \\
\hline 1 & 1794.2 & 1.8 & 1794.0 & 2.30 & 2.19 & -0.6820 & 0.2482 \\
2 & 2144.5 & 1.6 & 2146.5 & 2.43 & 2.26 & 6.0127 & $>0.9999$ \\
3 & 4748.8 & 3.6 & 4731.0 & 4.65 & 4.42 & -26.2133 & $<0.0001$ \\
4 & 7842.0 & 5.8 & 7831.8 & 10.07 & 9.21 & -7.5716 & $<0.0001$ \\
5 & 8622.9 & 5.5 & 8555.7 & 11.88 & 10.68 & -44.4345 & $<0.0001$ \\
6 & 16935.6 & 22.5 & 16506.8 & 19.61 & 20.36 & -124.4184 & $<0.0001$ \\
7 & 11849.4 & 5.6 & 11845.0 & 8.55 & 7.93 & -3.7282 & 0.0001 \\
8 & 16118.6 & 13.0 & 15756.6 & 23.10 & 21.08 & -118.2995 & $<0.0001$ \\
9 & 22800.2 & 18.4 & 22607.9 & 88.72 & 77.63 & -18.3804 & $<0.0001$ \\
10 & 18471.2 & 15 & 18442.1 & 12.04 & 12.83 & -13.1112 & $<0.0001$ \\
11 & 38211.8 & 28.7 & 37151.7 & 28.67 & 28.68 & -226.3141 & $<0.0001$ \\
\hline
\end{tabular}

\section{Research in perspective}

While this research presents a technique to obtain production sequences to optimize the even distribution of tool wear across multiple tooling sources, there are also limitations, which present opportunities for subsequent research.

\subsection{Benefit of research}

This research has presented a methodology for sequencing production to optimize the even distribution of tool wear across multiple sources. This sequencing technique consistently results in fewer tooling replacements compared with the previously published efforts.

\subsection{Limitations of research}

The above benefit most certainly has value - even distribution of tool wear, and the subsequent benefit of fewer tooling replacements is something that the production scheduler should seriously consider. Unfortunately, there is no reason to expect a 'direct' relationship between the tool wear measure $(W)$ and the expected number of replacements. While past research has demonstrated that even distribution of tool wear and expected number of replacements have a positive relationship to each other (a pilot study suggests a correlation of about $25 \%$, with $p$-values of linear association $<0.001$ ), there is no reason to believe that these values exist in perfect correlation. Subsequently, it is possible to obtain a production sequence with a minimal tool wear measure $(W)$ having an expected number of replacements inferior to that of another sequence with a suboptimal wear measure $(W)$. In fact, this was precisely what occurred with problem 2 of the problem set.

\subsection{Opportunities for subsequent research}

Given the presence of the above limitation, the author considers it prudent to further investigate the relationship between any measure of tool wear distribution and its 
relationship with expected replacements. There may in fact be objective measures that are better predictors of required tooling replacements. If an investigator can directly ascertain the relationship between a production sequence and its expected number of tooling replacements, the finding would have profound value.

It is also appropriate to note that the chosen heuristic approach to address this problem was simulated annealing. Simulated is one of many possible heuristics that can be used for problems of this type. Artificial neural networks, genetic algorithms, tabu search and other approaches could be used as well. These other potential approaches, and their associated CPU requirements, are mentioned here as an opportunity for further research.

\section{References}

Ding, F. and Cheng, L., An effective mixed-model assembly line sequencing heuristic for Just-in-time production systems. J. Op. Manage., 1993, 11, 45-50.

Eglese, R.W., Simulated annealing: a tool for operational research. Euro. J. Op. Res., 1990, 46, 271-281.

Fourer, R., Gay, D.M. and Kernighan, B.W., AMPL: A Modelling Language for Mathematical Programming, 2002 (Duxbury Press: Cincinnati, OH).

Kirkpatrick, S., Gelatt, C.D. and Veechi, M.P., Optimisation by simulated annealing. Science, 1983, 220, 671-679.

Kubiak, W. and Sethi, S., A note on 'level schedules for mixed-model assembly lines in just-intime production systems'. Management Science, 1991, 37, 121-122.

McMullen, P.R., Clark, M., Bell, J. and Albritton, D., A correlation and heuristic approach to production sequences with uniformity of tool wear. Comp. \& Op. Res., 2002, 30, 435-454.

McMullen, P.R., Sequencing for minimal tooling replacements via a variety of objective functions. Int. J. Prod. Res., 2003, 41, 2183-2199.

Metropolis, N., Rosenbluth, A., Rosenbluth, N., Teller, A. and Teller, E., Equation of state calculations by fast computing machines. J. Chem. Phys., 1953, 21, 1087-1092.

Miltenburg, J., Level schedules for mixed-model assembly lines in just-in-time production systems. Manage. Sci., 1989, 35, 192-207.

Nemhauser, G.L. and Wolsey, L.A., Integer and Combinatorial Optimisation, 1988 (John Wiley \& Sons: New York, NY).

Pinedo, M., Scheduling: Theory, Algorithms and Systems, 2001 (Prentice-Hall: Englewood Cliffs, NJ). 